Portland State University

PDXScholar

\title{
Localization of a Noisy Broadband Surface Target Using Time Differences of Multipath Arrivals
}

John Gebbie

Portland State University

Martin Siderius

Portland State University, siderius@pdx.edu

Reid McCargar

John Hopkins University, reid.mccargar@jhuapl.edu

John S. Allen III

University of Hawaii at Manoa

Grant Pusey

University of Hawaii at Manoa

Follow this and additional works at: https://pdxscholar.library.pdx.edu/ece_fac

Part of the Electrical and Computer Engineering Commons Let us know how access to this document benefits you.

\section{Citation Details}

Gebbie, J., Siderius, M., McCargar, R., Allen, J. S., \& Pusey, G. (2013). Localization of a noisy broadband surface target using time differences of multipath arrivals. Journal Of The Acoustical Society Of America, 134(1), EL77-EL83.

This Article is brought to you for free and open access. It has been accepted for inclusion in Electrical and Computer Engineering Faculty Publications and Presentations by an authorized administrator of PDXScholar. Please contact us if we can make this document more accessible: pdxscholar@pdx.edu. 


\title{
Localization of a noisy broadband surface target using time differences of multipath arrivals
}

\author{
John Gebbie, Martin Siderius, ${ }^{\text {a) }}$ and Reid McCargar ${ }^{\text {b) }}$ \\ Northwest Electromagnetics and Acoustics Research Laboratory, Department of Electrical \\ and Computer Engineering, Portland State University, 1900 Southwest 4th Avenue, \\ Suite 160, Portland, Oregon 97201 \\ jgebbie@ece.pdx.edu,siderius@pdx.edu,reid.mccargar@jhuapl.edu \\ John S. Allen III and Grant Pusey \\ Department of Mechanical Engineering, University of Hawaii-Manoa, 2540 Dole Street, \\ Holmes Hall 302, Honolulu, Hawaii 96822 \\ alleniii@hawaii.edu,grant.pusey@gmail.com
}

\begin{abstract}
Previous studies [Tiemann et al., J. Acoust. Soc. Am. 120, 2355-2365 (2006)] have reported the localization of marine mammals in 3-D from their clicks using multipath arrivals. Bathymetric variations were advantageously used to predict multipath arrival times with a raytracer. These arrivals are directly discernible from the time series for impulsive sources, such as whale clicks, but extension of the method to continuous broadband sources presents additional complications. By pulse compressing noise emitted from a small boat using two hydrophones, the hyperbolic direct-arrival ambiguity can be refined in both range and bearing. Acoustic-derived results are validated with target GPS measurements.
\end{abstract}

(C) 2013 Acoustical Society of America

PACS numbers: 43.30.Zk, 43.30.Cq, 43.30.Wi [AL]

Date Received: February 11, 2013 Date Accepted: May 23, 2013

\section{Introduction}

Passive acoustic methods have been shown to be a viable approach for small boat localization. ${ }^{1}$ These methods have some advantages over shore-based radar, infrared, and optical systems in that they are more robust to inclement weather and have the ability to be deployed in remote locations. They also have less impact on marine life than active sonar methods. Determining optimal techniques (e.g., number of sensors, layout of sensors, processing algorithms) for applying passive sonar in cluttered, shallow water environments, such as harbors, is an active area of research.

This paper presents a model-based localization technique that leverages reflections from a varying-bathymetry environment to refine estimates of the range and bearing to a small boat using two hydrophones. Multipath (or multipath arrivals) refers to echoes of a target's radiated sound from the surface and seabed. This technique differs from conventional hyperbolic fixing with passive sonar in which multipath arrivals are undesirable. Hyperbolic fixing involves measuring the time difference of arrival (TDOA) between pairs of distributed receivers. The TDOA between each receiver pair manifests spatially as a hyperbolic uncertainty region at the ocean surface as illustrated in Fig. 1(a). Localization is performed by finding intersections of multiple hyperbolae using additional hydrophone pairs. However, this is complicated by the fact that two hyperbolae can intersect at as many as four distinct points. ${ }^{2}$ This paper

\footnotetext{
a) Author to whom correspondence should be addressed.

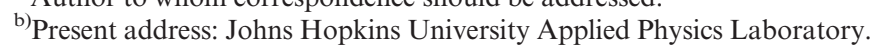



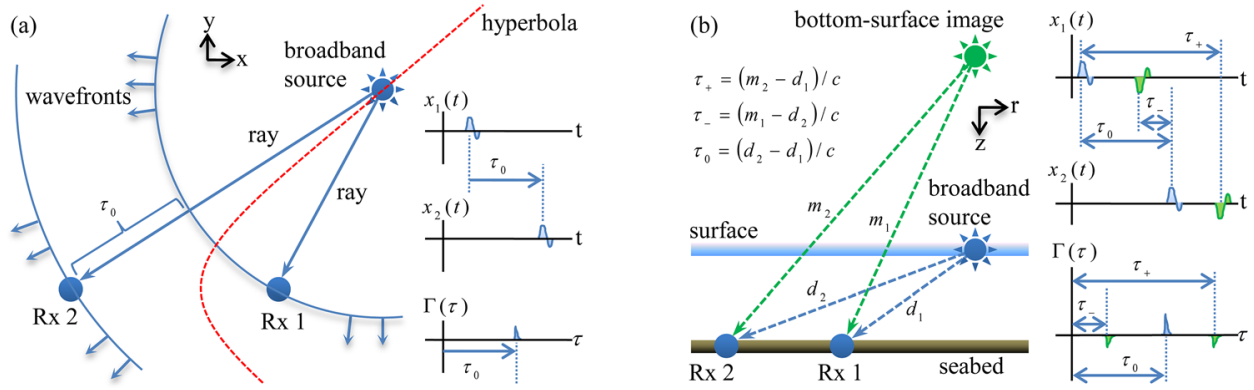

Fig. 1. (Color online) (a) Hyperbolic uncertainty (dashed line) from a single TDOA measurement. (b) Manifestation of TDOA $\left(\tau_{0}\right)$ and TDOMA $\left(\tau_{ \pm}\right)$for the first multipath arrival in a cross-correlation time series.

experimentally demonstrates that in an environment that produces multipath, the arrival information available at just two hydrophones encodes sufficient information to provide reasonable estimates of the range of a small boat. Further, varying bathyemtry can be opportunistically utilized to refine bearing.

Multipath information has been previously used for localization in several contexts. Phelan et al. (2012) demonstrated localization of a mobile radio transmitter in a complex urban environment using a two-dimensional (2-D) array by matching multipath arrival angles and delays with modeled values from a raytracer. ${ }^{3}$ In underwater acoustics, theoretical studies have shown that multipath information can provide improved range estimation of an underwater target compared to techniques that only utilize wavefront curvature. ${ }^{4}$ Blanc-Benon (1995) compared the use of time differences of multipath arrivals (TDOMA) received by an array with matched field processing on the same array and found that time differences were less sensitive to modeling errors. ${ }^{5}$ Recently there has been interest in using more cost-effective deployments consisting of only a few distributed sensors for marine mammal localization. It has been shown that whales, which use broadband clicks for echolocation, can be localized by measuring the TDOMA between the direct and bottom or surface-reflected paths to estimate range and depth. ${ }^{6,7}$ Tiemann et al. (2006) further demonstrated that only a single hydrophone was needed to produce a full 3-D localization of a sperm whale by comparing measured and modeled TDOMA using a raytracer. ${ }^{8}$ In that work, multipath arrivals were determined directly from the received time series (after some filtering) by time-gating each whale click and stacking the resulting snapshots with the direct arrivals vertically aligned. Viewed in this manner, all arrivals appeared as a time-evolving striation lines with multipath striations curving over time as the animal maneuvered relative to the hydrophone. ${ }^{9}$ Variations in bathymetry were opportunistically utilized to provide range, depth, and azimuthal discrimination by leveraging the fact that the relative arrival time of bottom-interacting multipath rays are functionally dependent on the bathymetry along each azimuthal radial.

Whale clicks are impulsive acoustic events that allow for clean separation of multipath arrivals in the received time series. However, the same does not hold for a continuous broadband noise source, such as that produced by a small boat. The technique proposed here uses pulse compression by cross-correlating two hydrophones allowing the same TDOMA quantities to be measured. In contrast to the scoring technique employed by Tiemann et al. (2006), a different ambiguity surface formulation is developed that projects multipath arrival times directly into the spatial domain. While TDOA (without multipath) produces two possible directions for the target, by using a single multipath arrival on each hydrophone, the range can also be estimated. Further, if the bathymetry has sufficient variability (and is known), the symmetry of the two possible directions can be broken, and a single direction and range can be determined. In the absence of multipath or sufficient bathymetry variation, the method degenerates to producing the TDOA hyperbolic surfaces. 
Experimental results from near Honolulu Harbor, HI, USA, compare localizations of a small boat with its GPS coordinate data.

\section{Multipath structure in shallow water}

In a simple passive sonar scenario characterized by an isovelocity environment with no shadow zones, the arrival structure consists of a single direct eigenray and a series of reflected eigenrays. The geometry of this problem is illustrated in Fig. 1(b) and shows two bottom-mounted hydrophones and a broadband target on the surface. The bottomsurface reflection appears as an image source located two water depths above the surface. The top two diagrammatic plots [Fig. 1(b), right] show the received time series, $x_{1}(t)$ and $x_{2}(t)$. The bottom plot shows the cross-correlation, $\Gamma(\tau)$. The source waveforms, $x_{1}(t)$ and $x_{2}(t)$ are represented as a single pulse for illustration purposes, but for a small boat target, they actually consist of continuous broadband noise. However, this does not affect the presence of pulses in $\Gamma(\tau)$ because the noise is pulse compressed through the cross-correlation operation. Environmental factors such as bottom loss and rough-surface scattering serve to decorrelate high-order eigenrays, whereas low-order eigenrays often retain enough coherence to appear as stable features in $\Gamma(\tau)$; therefore, only the first-order multipath arrival is shown in this illustration.

Because receiver 2 is farther from the source than receiver 1, both peaks in $x_{2}(t)$ are shifted later in time to account for the additional travel time. The term $\tau_{0}$ denotes the TDOA, and $\tau_{ \pm}$are the TDOMA. In $\Gamma(\tau)$, the strongest peak is in the center, with an absolute offset at $\tau_{0}$. The flanking (TDOMA) peaks are produced by the direct arrival from one hydrophone correlating with the multipath arrival from the other hydrophone. As the target initially moves into the far field of the hydrophone pair, $\tau_{-}$and $\tau_{+}$start to converge but are sufficiently large that the flanking peaks are distinct from the TDOA peak, but this separation eventually vanishes in the distant far field. Bathymetric variations affect $\tau_{ \pm}$because the eigenray path length depends on the depth of each bottom reflection, and this allows for bearing disambiguation.

\section{Localization algorithm}

The localization algorithm consisted of the following parts: The cross-correlation function, the multipath extraction, the ray model, and the ambiguity function. The crosscorrelation function, $\Gamma(\tau)$, was computed from a snapshot of two channels by the following procedure. Each channel was zero-meaned, windowed using a Hann function, passed through an FFT, and pre-whitened. Pre-whitening preserves phase information while enforcing a flat power spectrum and is defined as $X(\omega) /|X(\omega)|$ for an input spectrum $X(\omega) .{ }^{10}$ The cross-correlation was then performed by multiplying one channel with the complex-conjugate of the result, then bandpass filtered. After passing through an IFFT, the envelope of the resulting time series was computed by $|x(t)+\mathcal{H}[x(t)]|$, in which $x(t)$ is the input time series and $\mathcal{H}$ is the Hilbert transform.

A correlogram was formed by stacking $\Gamma(\tau)$ from each snapshot vertically. Viewed in this manner, striation lines appeared that corresponded to correlations of individual arrivals as they evolved over time. The center striation, which had an offset of $\tau_{0}$ for a given snapshot, was often the strongest as it corresponded to correlations of the direct arrivals on each hydrophone. The nearest flanking striations were the first multipath arrival correlating with the direct arrival, and had offsets $\tau_{ \pm}$for a given snapshot. Extraction of the points was facilitated through a graphical plotting routine in MATLAB. Linear interpolation resulted in a value of each striation at each snapshot. This manual process ensured the striations were extracted accurately and not subject to errors introduced by secondary extraction algorithms, thus providing an upper bound on the algorithm performance.

Predictions of eigenray propagation-time differences, $\bar{\tau}_{0}(\mathbf{x}), \bar{\tau}_{-}(\mathbf{x})$, and $\bar{\tau}_{+}(\mathbf{x})$, were computed for each possible (Easting, Westing) target position, $\mathbf{x}$, assuming a non-refracting medium. The bottom-surface image position was determined geometrically 
using a bathymetry database. The variables $\bar{\tau}_{ \pm}(\mathbf{x})$ are the predicted propagation-time differences between the direct and multipath eigenrays received at opposing hydrophones.

With the extracted arrival times, the correlogram was de-noised by reconstructing each snapshot using a sum-of-Gaussians as

$$
\hat{\Gamma}(\tau)=\alpha \sum_{\tau^{\prime} \in\left\{\tau_{0}, \tau_{-}, \tau_{+}\right\}} e^{-(1 / 2)\left[\left(\tau-\tau^{\prime}\right) / \sigma\right]^{2}} .
$$

In $\Gamma(\tau)$, the pulse corresponding to the pulse-compressed noise has a full width that is inversely proportional to the bandwidth of the target, $b$. So $\sigma$ was defined as $1 /(2 b)$, as $\sigma$ specifies the half-width of the Gaussian function. The term $\alpha$ normalizes $\hat{\Gamma}(\tau)$ to have range $[0,1]$.

The ambiguity function is defined as

$$
\Phi(\mathbf{x})=e^{-(1 / 2)\left\{\left[\tau_{0}-\bar{\tau}_{0}(\mathbf{x})\right] / \sigma\right\}^{2}} \cdot \hat{\Gamma}\left[\bar{\tau}_{-}(\mathbf{x})\right] \cdot \hat{\Gamma}\left[\bar{\tau}_{+}(\mathbf{x})\right] .
$$

The first term constrains the target location to the hyperbola determined by the TDOA of the direct arrivals. The latter two terms interpolate the value of $\hat{\Gamma}(\tau)$ at each $\bar{\tau}_{ \pm}$and assume large relative values when $\mathbf{x}$ matches the actual position of the target. Values of $\Phi(\mathbf{x})$ are in the range $(0,1]$ and yield information about the relative certainty of the target being at a particular location, $\mathbf{x}$, on the water surface.

\section{Experiment}

Passive acoustic signals were collected in August 2011 by a moored horizontal line array (HLA) at the Kilo Nalu Nearshore Reef Observatory. The observatory provided power and ethernet connectivity via an undersea cable running approximately $0.4 \mathrm{~km}$ from shore to a fixed underwater station deployed in roughly $12 \mathrm{~m}$ of water. The station was located about $1 \mathrm{~km}$ southeast of Honolulu Harbor, a commercial port. Only two elements of the HLA, spaced $11 \mathrm{~m}$ apart, were used in this study. The array was configured with a sample rate of $102.4 \mathrm{kHz}, 24$-bit dynamic range, $300 \mathrm{~Hz}$ low-cut filter, and $110 \mathrm{~dB}$ anti-aliasing filter set at $46.4 \mathrm{kHz}$. The hydrophones (HTI-92-WB) had a sensitivity of $-160 \mathrm{~dB}$ re $1 \mathrm{~V} / \mu \mathrm{Pa}$.

A rigid-hulled small boat with a single outboard engine was used as a target, and a handheld GPS device recorded its location. Time stamps in the GPS data and recorded acoustic data allowed for coarse-grained synchronization (on the order of $1 \mathrm{~s}$ ) between the two sets of data. The boat executed several different maneuvers including driving in circles around the array deployment site. Spectral analysis indicated that the boat radiated noise in the $0-10 \mathrm{kHz}$ band with the bulk of the energy below $3 \mathrm{kHz}$.
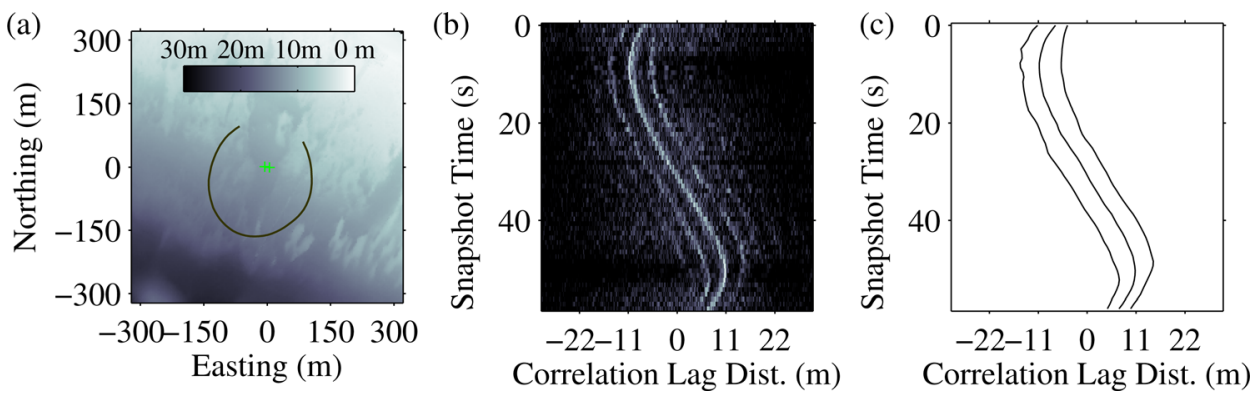

Fig. 2. (Color online) (a) Bathymetry and GPS boat track. The solid line shows the track of the small boat with a counter-clockwise trajectory. The "+" annotations indicate array element locations. (b) Correlogram showing $10 \log _{10}|\Gamma(\tau)|^{2}$ evolving over snapshot time, plotted using $30 \mathrm{~dB}$ of dynamic range and with $\tau$ converted to distance. (c) Striation lines for the TDOA $\left(\tau_{0}\right)$ and TDOMA $\left(\tau_{ \pm}\right)$, determined manually. These values facilitate denoising the correlogram, in which they serve as the parameters to $\hat{\Gamma}(\tau)$ defined in Eq. (1). 
(a) TDOA only

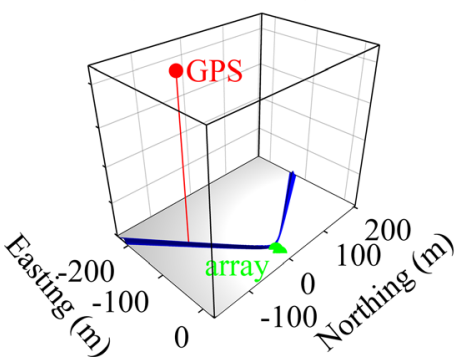

(b) Multipath, flat bottom

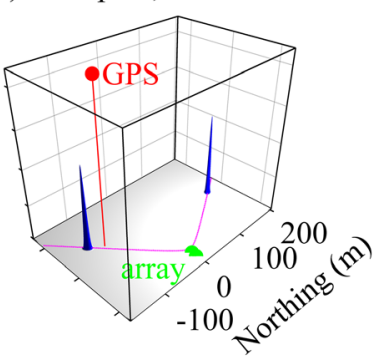

(c) Multipath, full bathy

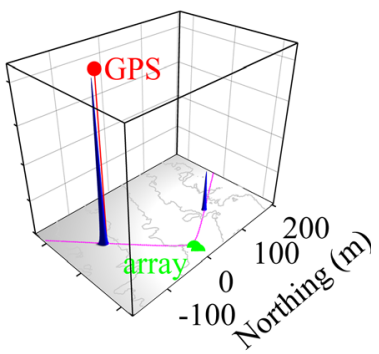

Fig. 3. (Color online) Comparison of ambiguity surfaces, $\Phi(\mathbf{x})$, for a single snapshot showing the effect of utilizing multipath and bathymetry information. Plots are normalized to unit volume to show the relative concentration of target location certainty. (a) A hyperbolic ambiguity is associated with only using the TDOA (first) term of Eq. (2). (b) Inclusion of multipath [the latter two terms of Eq. (2)] and the assumption of a flat seabed cause the hyperbola to collapse to a single range, but a left-right ambiguity remains. (c) Using actual bathymetry to determine the position of the bottom-surface image moves the range estimate on the near side closer to the GPS measurement and allocates a greater amount of target location certainty to it than the ambiguous peak. Contour lines are shown at $2.5 \mathrm{~m}$ intervals. For (b) and (c), the hyperbola defined by the TDOA, $\tau_{0}$, is also shown.

Bathymetry information for the local area was obtained from the SHOALS LIDAR bathymetry database at the University of Hawaii, ${ }^{11}$ which was ungridded data having roughly $1 \mathrm{~m}$ resolution. Grab samples near the deployment site indicated the seabed was composed of medium/coarse sand. The bathymetry, hydrophone locations, and track of the boat are shown in Fig. 2(a).

\section{Results}

A correlogram is shown in Fig. 2(b) in which multipath effects are evident. The strong, center striation is the correlation of direct arrivals. This is supported by the fact that as the target circles around the array, this striation stays between $\pm 11 \mathrm{~m}$, which are the limits for the correlation lag distance for the configured hydrophone spacing of 11 $\mathrm{m}$. The multipath-with-direct correlations are visible as "shadow" striations that run adjacent to the main striation. All these striation lines were manually traced using MATLAB and are shown in Fig. 2(c). Shadow striations from higher-order eigenrays are also faintly visible throughout the entire run.

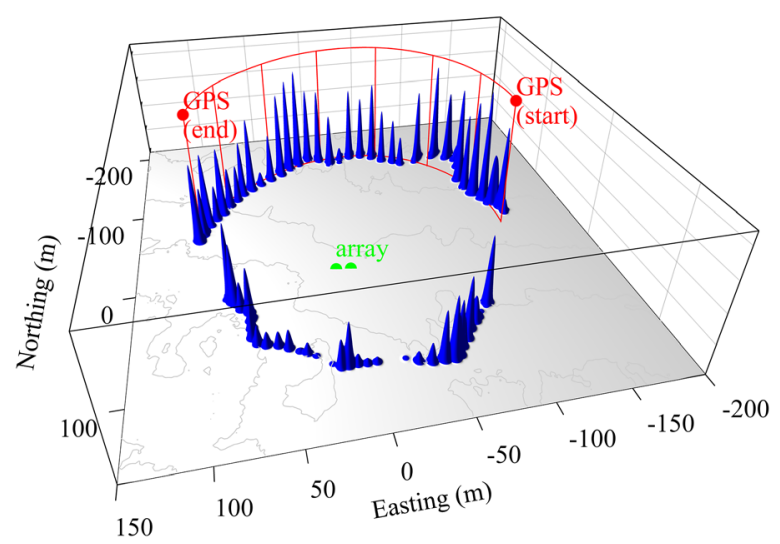

Fig. 4. (Color online) Ambiguity surfaces, $\Phi(\mathbf{x})$, for the sequence of snapshots between 13.5 and $47.5 \mathrm{~s}$ from the track shown in Figs. 2(a)-2(c). This period corresponds to when the boat is to the south of the array and sweeping through bearing angles from end-fire to end-fire. The camera is pointed mainly southward. The peaks on the far side of the array (where the boat is located) track well with the GPS data and have a higher certainty score than the peaks on the near side. 
A comparison of localization using only TDOA [corresponding to using just the first term of Eq. (2)] with both TDOA and TDOMA [all terms of Eq. (2)] is shown in subplots (a) and (b) of Fig. 3. The effects of using a flat seabed versus actual bathymetry are shown in subplots (b) and (c). The full ambiguity function, $\Phi(\mathbf{x})$, is shown for several snapshots throughout the boat track in Fig. 4. The image data in Figs. 3 and 4 were post-processed with a 2-D Hann filter to aid visualization of narrow features.

\section{Conclusion}

This paper presents a technique for localizing a small boat using multipath arrivals recorded on two bottom-mounted hydrophones. The correlations necessary to perform this inversion come from the lowest-order eigenrays, which are shown to be relatively stable features in a correlogram. Range information can be extracted from these features using image positions to estimate path length differences between direct and multipath eigenrays. Use of a bathymetry database for multipath ray calculation improves range localization and diminishes the left-right ambiguity typically associated with line arrays, but this is only possible in the presence of bathymetric variations. This experiment shows localization out to roughly 14 water depths, which would correspond to a much longer range in deeper water. Experimental results from passive acoustic measurements of a small boat maneuvering in a shallow-water harbor environment were validated by comparison with the boat's GPS $\log$.

This algorithm operates on a single snapshot but relies on accurate estimates of multipath correlation times, which were determined visually by examining the snapshot history. Tracking algorithms could be employed to provide these estimates either by following striations on the correlogram or by directly modeling the physics of the target. For snapshots in which multipath arrivals are not resolvable due to decorrelation or a longer distance target, this algorithm reverts to the standard TDOA-based hyperbolic ambiguity. Some additional factors that may impact performance include the source bandwidth, water depth, separation of sensors, sediment properties, and boundary roughness as well as Doppler effects. Future work can focus on generalization of this algorithm to underwater targets, alternate sensor configurations as well as an analysis of factors affecting performance. This is a preliminary study of multipathbased localization using two hydrophones, and improved results are expected by incorporating additional hydrophones, such as from a full array.

\section{Acknowledgments}

The authors acknowledge support for this research by the Office of Naval Research for the development of the localization algorithm as well as J.G.'s fellowship. We thank the Department of Homeland Security for sponsoring J.S.A. and for the experimental deployment under Grant Award 2008-ST-061-ML0001. We also thank Portland State University for funding. Additionally, we thank the Kilo Nalu Nearshore Reef Observatory and Tom Monroe of High Tech, Inc., for technical support.

\section{References and links}

${ }^{1}$ M. Bruno, K. W. Chung, H. Salloum, A. Sedunov, N. Sedunov, A. Sutin, H. Graber, and P. Mallas, "Concurrent use of satellite imaging and passive acoustics for maritime domain awareness," in IEEE International Waterside Security Conference (WSS) (2010), pp. 1-8.

${ }^{2} \mathrm{R}$. Schmidt, "A new approach to geometry of range difference location," IEEE Aerosp. Electron. Syst. 8, 821-835 (1972).

${ }^{3}$ B. R. Phelan, E. H. Lenzing, and R. M. Narayanan, "Source localization using unique characterizations of multipath propagation in an urban environment," in IEEE 7th Sensor Array and Multichannel Signal Processing Workshop (SAM) (2012), pp. 189-192.

${ }^{4}$ M. Hamilton and P. M. Schultheiss, "Passive ranging in multipath dominant environments. I. Known multipath parameters," IEEE Trans. Signal Process. 40, 1-12 (1992). 
${ }^{5}$ P. Blanc-Benon, "Implication of shallow waters for source localization: Time-delays estimation versus matched-field processing," in OCEANS'95. MTS/IEEE. Challenges of Our Changing Global Environment. Conference Proceedings (1995), Vol. 2, pp. 826-831.

${ }^{6}$ R. Aubauer, M. O. Lammers, and W. W. L. Au, "One-hydrophone method of estimating distance and depth of phonating dolphins in shallow water," J. Acoust. Soc. Am. 107, 2744-2749 (2000).

${ }^{7}$ E. Nosal and L. Neilfrazer, "Track of a sperm whale from delays between direct and surface-reflected clicks," Appl. Acoust. 67, 1187-1201 (2006).

${ }^{8}$ C. O. Tiemann, A. M. Thode, J. Straley, V. O'Connell, and K. Folkert, “Three-dimensional localization of sperm whales using a single hydrophone," J. Acoust. Soc. Am. 120, 2355-2365 (2006).

${ }^{9} \mathrm{C}$. O. Tiemann, "Three-dimensional single-hydrophone tracking of a sperm whale demonstrated using workshop data from the Bahamas," J. Can. Acoust. Assoc. 36, 67-73 (2008).

${ }^{10}$ G. C. Carter, A. H. Nuttall, and P. G. Cable, "The smoothed coherence transform," Proc. IEEE 61, 1497-1498 (1973).

${ }^{11}$ University of Hawaii at Manoa, Costal Geoglogy Group, SHOALS LIDAR Bathymetry Database (2012), www.soest.hawaii.edu (Last viewed April 2012). 